\title{
Multitarget drugs: Focus on the NO-donor hybrid drugs*
}

\author{
Alberto Gasco $\ddagger$, Donatella Boschi, Konstantin Chegaev, Clara Cena, \\ Antonella Di Stilo, Roberta Fruttero, Loretta Lazzarato, \\ Barbara Rolando, and Paolo Tosco
}

\author{
Department of Pharmaceutical Science and Technology, University of Turin, \\ via P. Giuria 9, I-10125 Turin, Italy
}

\begin{abstract}
The article addresses the design of multitarget drugs, namely, compounds capable of interacting with more than one target simultaneously. These agents could be useful tools in the therapy of complex diseases such as cardiovascular and inflammatory diseases. An interesting case of multitarget compounds are nitric oxide (NO)-donor hybrids, structures which combine the physiological properties of $\mathrm{NO}$ with those of a lead drug. In particular, the authors discuss the symbiotic approach used to design NO-donor nonsteroidal anti-inflammatory drugs (NO-NSAIDs) and NO-donor antioxidants. The former could be useful agents in the treatment of anti-inflammatory diseases being devoid of gastro- and cardiotoxicity, the latter could be a valid approach to the treatment of many cardiovascular diseases.
\end{abstract}

Keywords: multitarget drugs; NO-donor hybrid drugs; NO-NSAIDs; NO-antioxidants; NO-donors; nitric oxide (NO).

\section{INTRODUCTION}

Most drugs commonly used in therapy have been developed on the basis of the reductionistic "one target-one disease" approach. They are able to address individual targets, and consequently they are successfully used in single-target therapy. They are also used in combination for the treatment of complex diseases, such as cardiovascular and inflammatory diseases, cancer, and AIDS, which require addressing more than one target. In this regard, today there is great interest in the use of multitarget drugs, also called polyvalent or multifunctional drugs, namely, single products capable of interacting simultaneously with multiple targets, directly or following metabolism [1,2]. The use of polyvalent drugs shows some advantages compared with a cocktail of drugs, including a lower risk of drug-drug interactions, improved compliance by the patient, and a more predictable pharmacokinetic profile. The major disadvantage is the difficulty to adjust the activities toward different targets in an appropriate ratio within a single chemical structure. There are two different approaches to designing a polyvalent drug, namely, the "symbiotic approach", also called "medicinal chemical hybridization" $(\mathrm{MCH})$, and the "screening approach" [1,2]. The symbiotic approach is an old technique in drug design [3-6], and it is carried out by fusing (fusion) or by joining through an appropriate linker (conjugation) two drugs or crucial parts of them. The linker can be either susceptible to metabolic cleavage or a hard linker. In some cases, it is possible to carry out this approach by overlapping substructures which are common to the two leads

\footnotetext{
*Paper based on a presentation at the $41^{\text {st }}$ IUPAC World Chemistry Congress, 5-11 August 2007, Turin, Italy. Other presentations are published in this issue, pp. 1631-1772.

${ }^{\ddagger}$ Corresponding author
} 
(merger). Recently, about 100 target combinations used to develop multitarget drugs were discussed [1]. The screening approach is carried out by testing through appropriate bioassays random libraries of products, focused libraries of products, or libraries of known drugs. Broadly speaking, a multifunctional drug obtained through these two approaches must undergo appropriate chemical modifications in order to both "balance" it and optimize its physicochemical properties [1,2,7]. The concept of balance is central in the multitarget approach. A polyvalent drug obtained by combining leads is well "balanced" when it displays the desired pharmacological actions of the leads at a similar efficacious dose. Generally, the symbiotic approach does not give rise to a well-balanced product, and so it is necessary to adjust its ratio of activities by changing its structure appropriately. This can be a very difficult challenge. Knowledge of structure-activity relationships (SARs) which operate in the lead drugs, as well as of the structures of the targets, is an invaluable aid in this task. When SARs are known totally or partly, they should drive both the appropriate way of combining the leads and the further structural modifications of the hybrid. If they are unknown, the approach is entirely left to the intuition and experience of the researcher.

\section{NO-DONOR HYBRID DRUGS}

An interesting case of multitarget compounds is that of nitric oxide (NO)-donor hybrids, namely, structures that combine the physiological properties of NO with those of a lead drug. NO is a physiological messenger which displays a variety of effects in the cardiovascular system, in the central and peripheral nervous systems. In particular, it dilates blood vessels, inhibits platelet adherence and aggregation, attenuates leukocyte adherence and activation, and inhibits vascular smooth muscle cell proliferation. It is also one of the final effectors of the immune system [8]. Consequently, today there is wide interest in products collectively called NO-donors, namely, in structures that are able to release NO in physiological solution [9]. A number of them are commonly used in therapy for the treatment of some cardiovascular disorders. The organic esters of nitric acid, the organic nitrates, are the most important among them. Glyceryl trinitrate (GTN) is the prototype of these important drugs. A NO-donor hybrid can be obtained by combining an established drug with an appropriate NO-donor through the aforementioned symbiotic approach. Nicorandil (Fig. 1) is an example of these products. It was designed by joining through a dimethylene bridge a nicotinamide substructure, able to open $\mathrm{K}^{+}$-channels, with an $O$-nitro function capable of releasing NO. This drug is commonly used to treat the pain associated with angina pectoris. In our laboratory, we designed a number of NO-donor hybrids $[10,11]$, and in our symbiotic approach we frequently used the furoxan system (Fig. 1) as NO-donor moiety. Furoxan (1,2,5-oxadiazole 2-oxide) [12] represents an important part of the chemical history of the University of Turin, since Prof. Giacomo Ponzio studied it at the Chemical Institute for many years during the first half of the $20^{\text {th }}$ century. In the recent past, it was found that furoxan derivatives are able to release NO when incubated in physiological solution in the presence of thiols [13]. Plasma components seem to potentiate this release [14]. The mechanism of the NO-donation is not yet fully understood and is in need of additional investigation. Since the capacity to release NO by the furoxan system can be modulated by changing the substituents at the ring and, in general, it is in-<smiles>O=C(NCCO[N+](=O)[O-])c1cccnc1</smiles>

Nicorandil

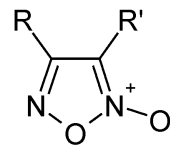

Furoxan

Fig. 1 Structure of Nicorandil and of the furoxan system. 
creased by the presence of electron-withdrawing groups, furoxan moieties appear to be flexible tools for balancing the designed hybrids [11,15].

Recently, we have been interested in two classes of NO-donor hybrids: NO-donor nonsteroidal anti-inflammatory drugs (NO-NSAIDs) and NO-donor antioxidants.

\section{NO-DONOR NONSTEROIDAL ANTI-INFLAMMATORY DRUGS}

NO-NSAIDs are an interesting class of products designed to combine the gastrosparing effects of NO with anti-inflammatory properties of NSAIDs [16]. Examples of these products are reported in Fig. 2. NO-aspirins are the most frequently studied among these compounds. They were originally obtained by NicOx Company by joining the $\mathrm{COOH}$ group of aspirin with moieties containing NO-donor $O$-nitro functions through a simple ester bridge. Prototypes of such structures are products NCX 4016 and NCX 4215 [17]. Subsequently, a second family of NO-donor aspirins was developed by our group [18]. They are structurally similar to the previous products but they contain furoxan derivatives as NO-donor substructures. More recently, a new class of NO-aspirins has been developed containing moieties in which the $-\mathrm{ON}=(\mathrm{O}) \mathrm{N}-\mathrm{N}<$ substructure linked to the carboxylic group through a methylene bridge is present [19]. These products give rise, by chemical and enzymatic metabolism, to free diazeniumdiolates, which are able to release NO spontaneously. Today, it is becoming more and more evident that these products display a number of effects that go beyond their original intended use [20]. They display a variety of actions, including anti-inflammatory and analgesic effects, antiplatelet and vasodilator properties, beneficial effects in the treatment of restenosis, myocardial ischemia, and infarction. Additionally, a number of them proved to be more potent than aspirin in inhibiting proliferation of several cancer cell lines, including prostate cancer cell systems. NCX 4016 was found to enhance the preventive and therapeutic effectiveness of the antitumor immunity elicited by cancer vaccination. Very recently, a potential indication of NO-aspirins in the treatment of type 2 diabetes has been proposed by NicOx Company [20]. The action mechanism of these products needs additional investigation, also considering that some
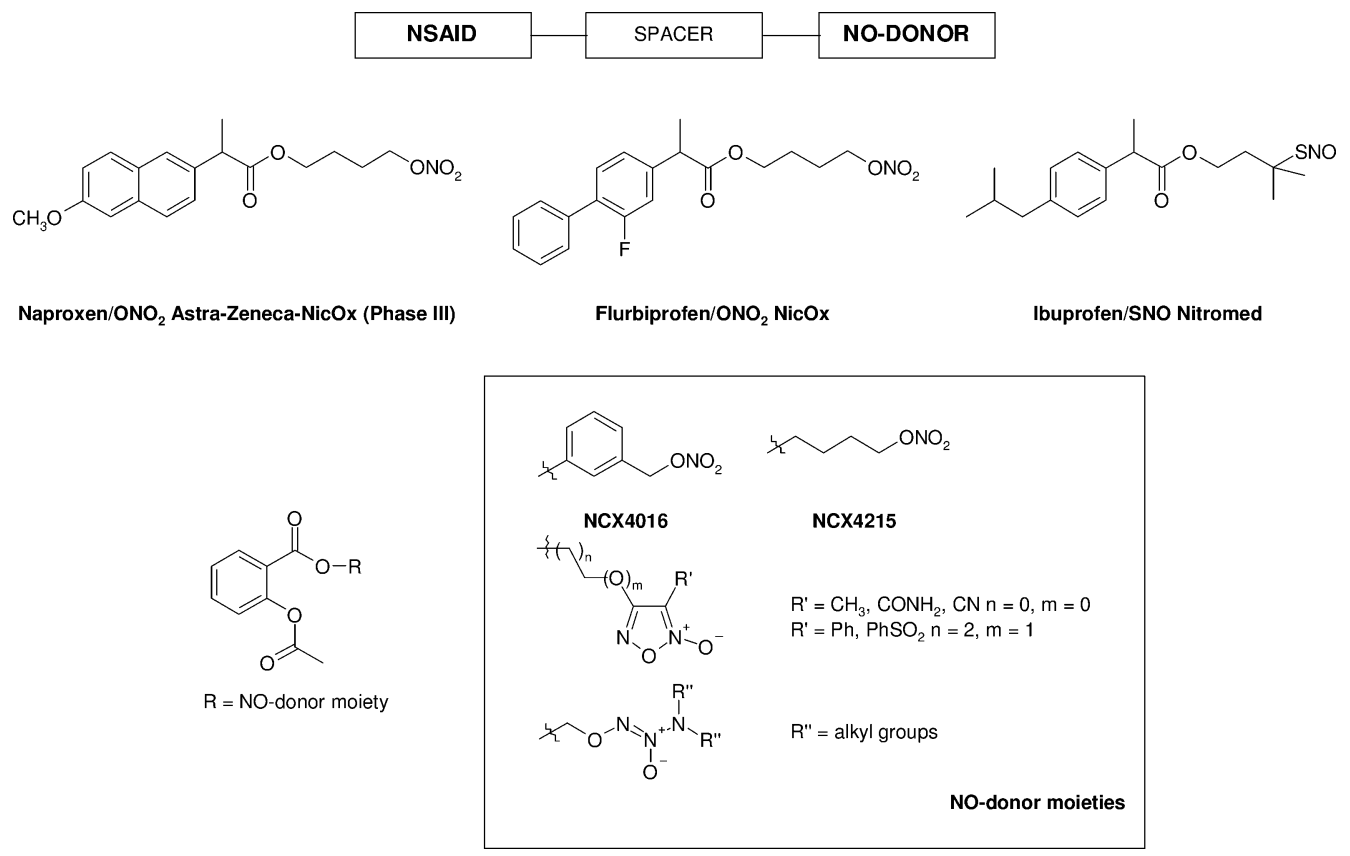

Fig. 2 Examples of NO-NSAIDs. 
of them, and in particular NCX 4016, one of the most widely studied products, are not true prodrugs of aspirin. Actually, when incubated in plasma and other physiological media, they are rapidly metabolized with little or no formation of aspirin [18,21].

As a development of our work in this area, we recently designed a new class of NO-donor aspirin-like drugs [22]. These products are formally derived from aspirin by linking acyl moieties bearing $\mathrm{NO}$-donor $O$-nitro groups to the $-\mathrm{OH}$ function of salicylic acid (Fig. 3). They are not prodrugs of aspirin, but true aspirin/NO-donor hybrids, in which the NO-donor moieties are linked to aspirin by a hard linker. All the products are largely stable over $3 \mathrm{~h}$ at $\mathrm{pH}=1$ and at physiological $\mathrm{pH}$. When incubated in serum the mono $O$-nitro derivatives (M1-M6) are principally metabolized by serum esterases to salicylic acid and to the corresponding $O$-nitro substituted alkanoic acids, with the only exception of M1 which affords a mixture of products. Their half-life times are dependent on the structure of the lateral chain (Table 1). As the chain is lengthened, they increase and decrease when $n$ is higher than 4 (M5). Dimethyl substitution at the carbon $\alpha$-positioned to the ester function definitely stabilizes the product (M6). Similar behavior is shown by the di $(O$-nitro) derivatives (D1-D5). When administered to rats by intragastric (i.g.) route at a dose equimolar with aspirin $(120 \mathrm{mg} / \mathrm{Kg}$ i.g.), after $3 \mathrm{~h}$ all the products display markedly less gastric damage compared with the lead. By contrast, many of the products trigger anti-inflammatory effects as potent as the ones displayed by aspirin when tested on carrageenan-induced paw edema in rat (Fig. 4). A number of these products were also able to inhibit platelet aggregation induced by collagen in human platelet-rich plasma (PRP) (Table 1). It is interesting to underline that this effect is not influenced by the presence of ODQ $(1 H$-[1,2,4]oxadiazolo[4,3a]quinoxalin-1-one), a well-known inhibitor of the soluble guanylate cyclase (sGC), and this means that it is independent of the activation of sGC. Preliminary results indicate that it is the result of COX inhibition. Due to the presence of $O$-nitro NO-donor moieties, all the compounds proved to relax rat aorta strips precontracted with phenylephrine. In contrast to the anti-aggregatory action, this effect is cGMP-dependent since the vasodilator potencies of the products strongly decreased in the presence of ODQ (Table 1).

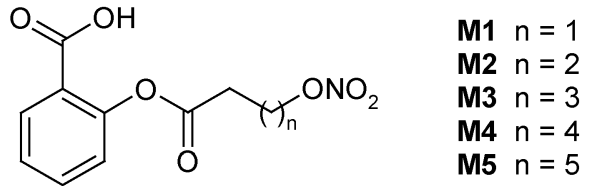<smiles>CC(C)(CO[N+](=O)[O-])C(=O)Oc1ccccc1C(=O)O</smiles>

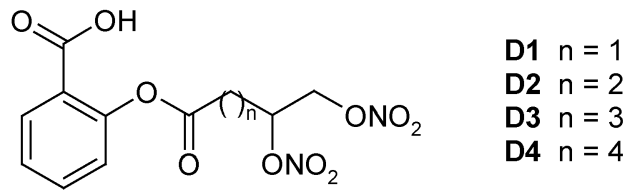<smiles>CC(C)(CCC(CO[N+](=O)[O-])O[N+](=O)[O-])C(=O)Oc1ccccc1C(=O)O</smiles>

Fig. 3 NO-donor aspirin-like compounds. 
Table 1 Stability in human serum, anti-aggregatory and vasodilator activity of the NO-donor "aspirin-like" compounds.

\begin{tabular}{lcccc}
\hline Compound & $\begin{array}{c}\text { Stability in } \\
\text { human serum } \\
t_{1 / 2}(\mathrm{~h})\end{array}$ & $\begin{array}{c}\text { Anti-aggregatory activity } \\
\mathrm{IC}_{50}(\mathrm{CL} 95 \%) \mu \mathrm{M} \\
+50 \mu \mathrm{M} \text { ODQ }\end{array}$ & $\begin{array}{c}\text { Vasodilator activity } \\
\mathrm{EC}_{50}(\mu \mathrm{M}) \pm \text { SEM }^{\mathrm{b}}\end{array}$ \\
\hline ASA & 1.06 & $54(49-60)$ & $/ / /$ \\
M1 & 0.26 & $162(129-204)$ & $186(172-200)$ & $37 \pm 2$ \\
M2 & 0.47 & $30(24-37)$ & $24(17-34)$ & $23 \pm 6$ \\
M3 & 0.76 & $97(85-110)$ & $91(76-110)$ & $21 \pm 2$ \\
M4 & 2.03 & $27 \pm 10^{\mathrm{a}}$ & & $14 \pm 1$ \\
M5 & 1.19 & Inactive & & $8.1 \pm 1.4$ \\
M6 & 9.03 & $26 \pm 6^{\mathrm{a}}$ & & $6.2 \pm 0.9$ \\
D1 & 0.28 & $126(98-160)$ & $119(105-134)$ & $9.2 \pm 0.9$ \\
D2 & 4.09 & $15 \pm 8^{\mathrm{a}}$ & & $8.2 \pm 1.2$ \\
D3 & 4.87 & $6.7 \pm 5.8^{\mathrm{a}}$ & & $5.8 \pm 0.7$ \\
D4 & 6.25 & Inactive & $3.1 \pm 0.7$ \\
D5 & 15.2 & Inactive & $5.3 \pm 0.6$ \\
\hline
\end{tabular}

aDue to the low activity of the compound, $\mathrm{IC}_{50}$ could not be calculated. In this case, the percent of inhibition \pm SEM at $300 \mu \mathrm{M}$ is reported.

${ }^{\mathrm{b}}$ In the presence of $1 \mu \mathrm{M}$ ODQ, $\mathrm{EC}_{50}$ values were $>100 \mu \mathrm{M}$.

\section{antiinflammatory activity}

carrageenan induced paw edema at $3 \mathrm{~h}$ in rat

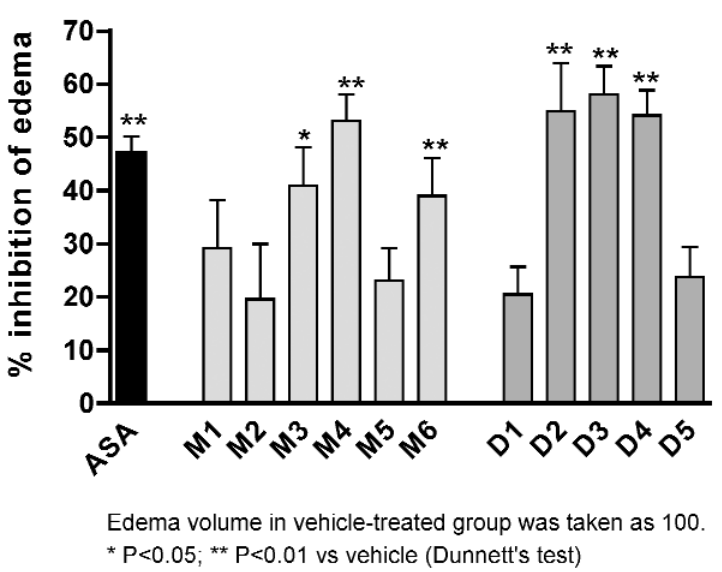

ulcerogenic effects

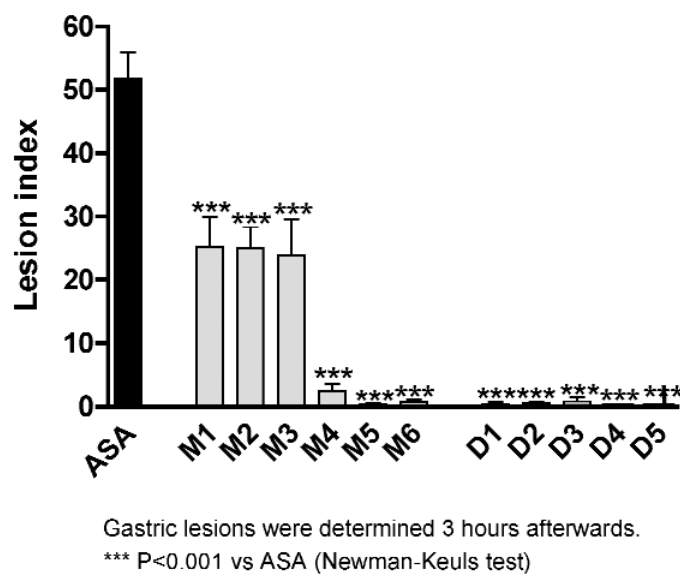

Fig. 4 Anti-inflammatory and gastrosparing properties of the NO-donor "aspirin-like" compounds.

\section{NO-DONOR ANTIOXIDANTS}

NO-donor antioxidants are a class of NO-donor hybrid drugs which today are the focus of great interest. Aerobic cells consume oxygen in oxidative reactions in order to produce energy. About $98 \%$ of the oxygen introduced into our organism is transformed into water. The remaining $2 \%$ is transformed into highly toxic and very reactive oxygen species collectively called ROS. When the balance between endogenous antioxidant defenses and ROS production is perturbed, a situation called oxidative stress arises. Oxidative stress is involved in many disorders, in particular, cardiovascular disorders and among them atherosclerosis. Atherosclerosis is a complex disease in which there is an accumulation of ROS and low-density lipoproteins (LDLs) in the vascular sub-endothelial space. This induces a high degree 
of lipid peroxidation and is an early stage of a complex process which leads first to the formation of foam cells, then of the fatty streak and ultimately of the atherosclerotic plaque [23]. There is a huge amount of experimental evidence that the administration of exogenous antioxidants can reduce the rate of formation of the atherosclerotic plaque in humans and animals [23]. Moreover, in an atherosclerotic vessel the ability of the endothelial cells to produce NO is reduced as is the sensitivity of the vessel to the action of $\mathrm{NO}$ [24], hence the interest in compounds deriving from the combination of an antioxidant structure with a NO-donor moiety. In our laboratory, we designed many of such compounds, including a new class of products in which selected phenols were conjugated to NO-releasing moieties [25]. The phenols used in our approach are characterized by having both $-\mathrm{OH}$ bond dissociation energies and lipophilicities widely modulated (Fig. 5). These molecular descriptors have a strong influence in determining the antioxidant profile of a phenol derivative. As NO-donor moieties, we chose the 3-carbamoylfuroxan and the 3-phenylsulfonylfuroxan substructures which are present in two orally active antihypertensive drugs developed by Cassella-Hoechst and Chiesi Company, respectively [26,27] (Fig. 5 ). We also addressed our attention toward the $O$-nitro function present in classical organic nitrates. All these reference NO-donor structures display widely modulated in vitro vasodilator properties. The products we obtained using the 3-phenylsulfonylfuroxan substructure proved to inhibit in a concentration-dependent manner the auto-oxidation of lipids present in microsomial membranes of rat hepatocytes, thiobarbituric acid reactive substances (TBARS) assay (Table 2). Moreover, in keeping with the presence in their structure of the phenylsulfonylfuroxan moiety, they proved to relax rat aorta strips precontracted with phenylephrine. This effect is cGMP-dependent since the vasodilator potencies of the products were strongly decreased in the presence of ODQ (Table 2). When the antioxidant and the vasodilator potencies of these products are compared $\left(\mathrm{IC}_{50} / \mathrm{EC}_{50}\right.$ ratio), it is possible to observe that all these products are, in vitro, unbalanced hybrids since their vasodilator properties prevail over the an-

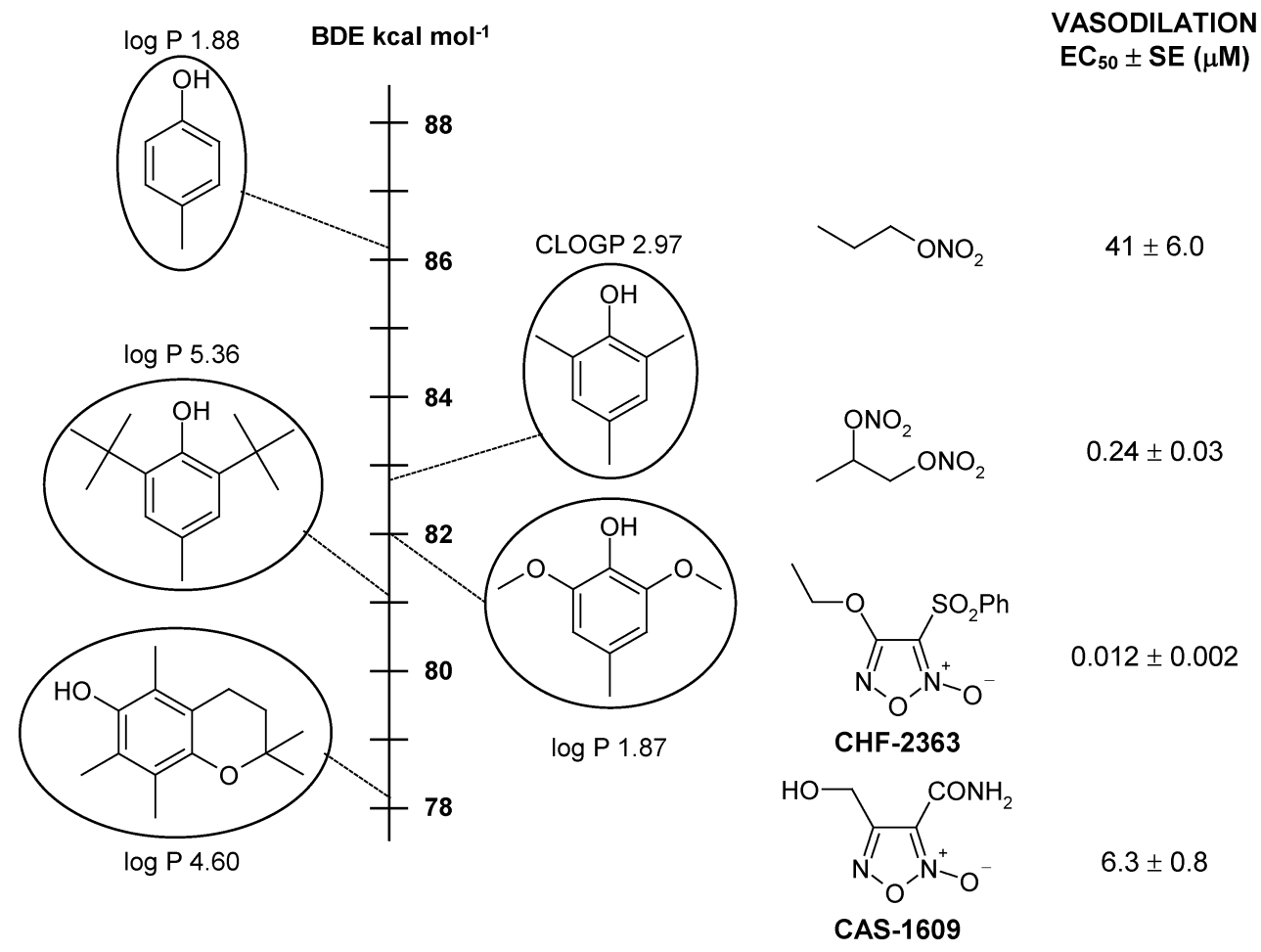

Fig. 5 Phenols and NO-donors used in the symbiotic approach to obtain NO-donor antioxidant hybrids. 
Table 2 Antioxidant and vasodilating activity of the NO-donor phenols.<smiles>[R]c1cc([R])c(O)c([R])c1</smiles>

A<smiles>[R]CC1(C)CCc2c(C)c(O)c(C)c(C)c2O1</smiles>

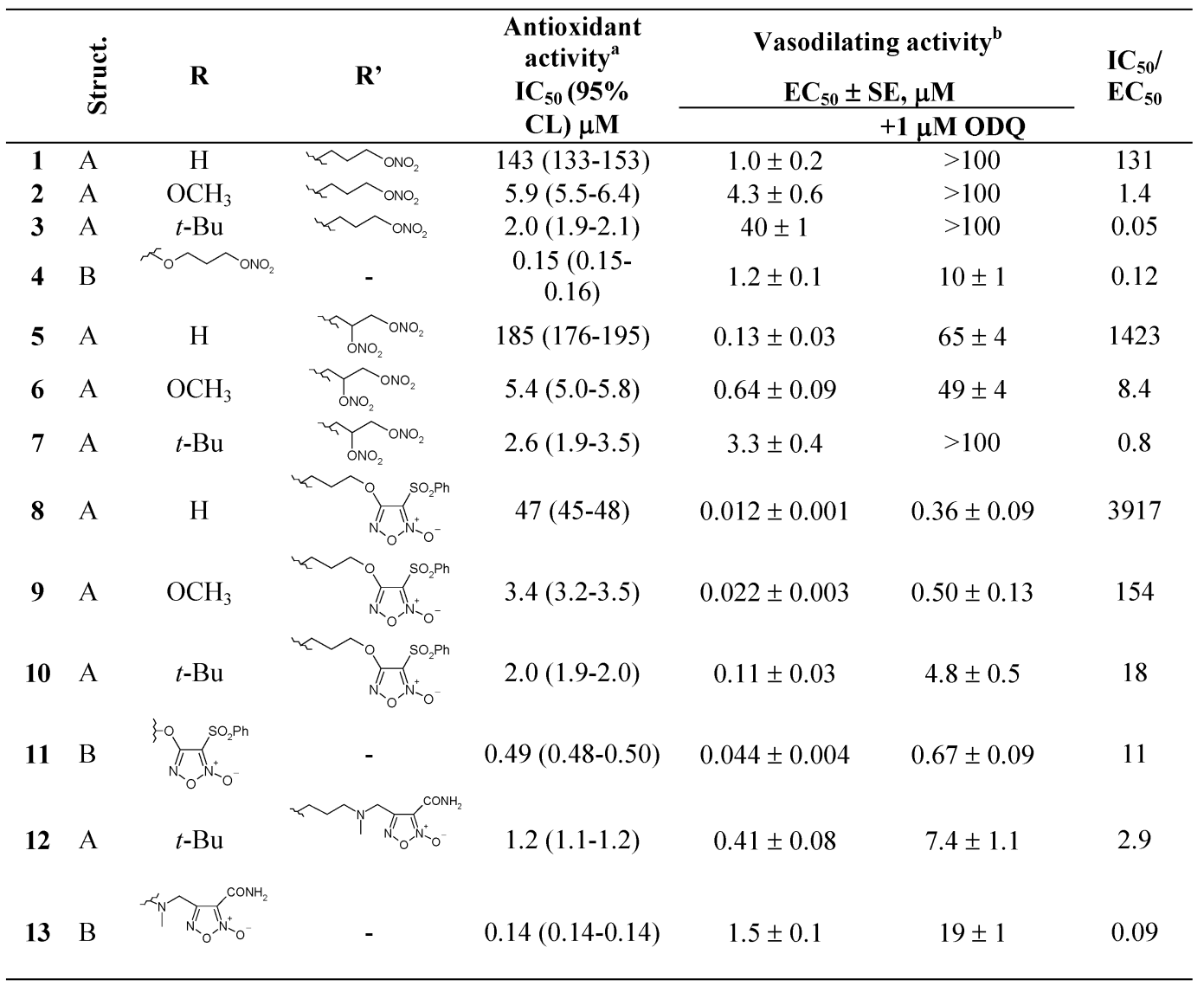

avalues are the means of at least five experiments.

${ }^{b}$ Values are the means of at least six experiments.

tioxidant ones (Table 2). Also, the products obtained using 3-carbamoylfuroxan and $O$-nitro moieties as NO-donors proved to display in vitro antioxidant and vasodilator properties. In this case, however, these two activities were modulated on a broad range, with the consequence that some products $(\mathbf{1}, \mathbf{5}, \mathbf{6}, \mathbf{8}-\mathbf{1 1})$ behaved principally as vasodilators, some $(\mathbf{3}, \mathbf{4}, \mathbf{1 3})$ as antioxidants and a few $(\mathbf{2}, \mathbf{7}, \mathbf{1 2})$ as well-balanced hybrids. A quantitative structure-activity relationship (QSAR) study shows that the antioxidant potencies are quite well described by a parabolic dependence on the lipophilicity $(\log P)$ and a linear dependence on the calculated $\mathrm{O}-\mathrm{H}$ bond dissociation energy $\left(\Delta H_{\text {abs }}\right)$ (eq. 1). The optimal $\log P$ is near 5.

$$
\begin{aligned}
& -\log \mathrm{IC}_{50}=-0.15( \pm 0.02) \Delta H_{\mathrm{abs}}-0.079( \pm 0.05)(\log P)^{2}+ \\
& 0.90( \pm 0.43) \log P+4.29( \pm 0.96) \\
& n=17, r^{2}=0.97, s=0.21, F=126.40, q^{2}=0.94
\end{aligned}
$$


Preliminary results show that a remarkable reduction in the extension of the infarct was observed when rat hearts previously subjected to ischemia were reperfused with a physiological solution containing selected members of this new series of NO-donor phenols in an appropriate concentration. An example is reported in Fig. 6.

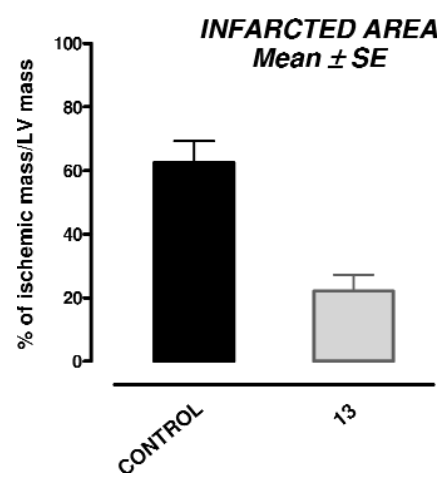

Fig. 6 Reduction in the extension of infarct in ischemia-reperfusion isolated rat heart when treated with $\mathbf{1 3}$ (1 $\mu \mathrm{M})$.

\section{CONCLUSIONS}

The multitarget approach in drug design is expected to give better results in the treatment of complex diseases than the use of cocktails of single-target drugs. NO-donor hybrids are a particular kind of such products which combine NO properties with those of an appropriate reference drug. Among them, NO-donor aspirins and NO-donor antioxidants have been an object of particular interest. The former could display aspirin properties without gastrotoxicity. The latter could be of interest in the treatment of some forms of cardiovascular disease, in particular, atherosclerosis, in view of their simultaneous ability to trigger beneficial NO-dependent effects on the cardiovascular system and antioxidant properties.

\section{ACKNOWLEDGMENTS}

This research was supported by a MIUR grant. The authors thank Profs. Gabriella Coruzzi and Giuseppina Morini of the Pharmacology Institute of the University of Parma for the in vivo studies.

\section{REFERENCES}

1. R. Morphy, C. Kay, Z. Rankovic. Drug Discov. Today 9, 641 (2004).

2. R. Morphy, Z. Rankovic. J. Med. Chem. 48, 6523 (2005) and refs. cited therein.

3. E. J. Ariëns. In Drug Design, E. J. Ariëns (Ed.), pp. 1-270, Academic Press, New York (1971).

4. J. J. Baldwin, W. C. Lumma, G. F. Lundell, G. S. Ponticello, A. W. Raab, E. L. Engelhardt, R. Hirschmann, C. S. Sweet, A. Scriabine. J. Med. Chem. 22, 1284 (1979).

5. B. J. Nicolaus. In Decision Making in Drug Research, F. Gross (Ed.), pp. 173-186, Raven Press, New York (1983).

6. J. A. M. Christiaans, H. Timmerman. Eur. J. Pharm. Sci. 4, 1 (1996).

7. R. Morphy, Z. Rankovic. J. Med. Chem. 49, 4961 (2006).

8. J. F. Kerwin Jr., R. Lancaster Jr., P. L. Feldman. J. Med. Chem. 38, 4343 (1995).

9. P. G. Wang, M. Xian, X. Tang, X. Wu, Z. Wen, T. Cai, A. J. Janczuk. Chem. Rev. 102, 1091 (2002) 
10. A. Gasco, R. Fruttero, B. Rolando. Mini Rev. Med. Chem. 5, 217 (2005).

11. A. Gasco, K. Schönafinger. In Nitric Oxide Donors, P. G. Wang, T. B. Cai, N. Taniguchi (Eds.), pp. 131-175, Wiley-VCH, Weinheim (2005).

12. A. Gasco, A. J. Boulton. Adv. Heterocycl. Chem. 29, 251 (1981).

13. M. Feelisch, F. Schönafinger, E. Noack. Biochem. Pharmacol. 44, 1149 (1992).

14. C. M. Turnbull, C. Cena, R. Fruttero, A. Gasco, A. G. Rossi, I. L. Megson. Br. J. Pharmacol. 148, 517 (2006).

15. A. Gasco, R. Fruttero, G. Sorba, A. Di Stilo, R. Calvino. Pure Appl. Chem. 76, 973 (2004).

16. U. K. Bandarage, D. R. Janero. Mini Rev. Med. Chem. 1, 57 (2001).

17. P. del Soldato, R. Sorrentino, A. Pinto. Trends Pharmacol. Sci. 20, 319 (1999).

18. C. Cena, M. L. Lolli, L. Lazzarato, E. Guaita, G. Morini, G. Coruzzi, S. P. McElroy, I. L. Megson, R. Fruttero, A. Gasco. J. Med. Chem. 46, 747 (2003).

19. C. Velázquez, P. N. P. Rao, E. E. Knaus. J. Med. Chem. 48, 4061 (2005).

20. C. M. Turnbull, A. G. Rossi, I. L. Megson. Expert Opin. Ther. Targets 10, 911 (2006).

21. J. J. Gao, K. Kashfi, R. Rigas. J. Pharmacol. Exp. Ther. 31, 989 (2005).

22. A. Gasco, R. Fruttero, L. Lazzarato, M. Donnola. WO2007060112.

23. J. F. Keaney Jr., J. A. Vita. Prog. Cardiovasc. Dis. 38, 129 (1995).

24. G. A. Dillon, J. A. Vita. In Nitric Oxide and the Cardiovascular System, J. Loscalzo, J. A. Vita (Eds.), pp. 207-226, Humana Press, Totowa, NJ (2000).

25. D. Boschi, G. C. Tron, L. Lazzarato, K. Chegaev, C. Cena, A. Di Stilo, M. Giorgis, M. Bertinaria, R. Fruttero, A. Gasco. J. Med. Chem. 49, 2886 (2006).

26. H. Bohn, J. Brendel, P. A. Martorana, K. Schönafinger. Br. J. Pharmacol. 114, 1605 (1995).

27. M. Civelli, M. Giossi, P. Caruso, R. Mazzetti, M. Bergamaschi, S. Bongrani, A. Gasco. Br. J. Pharmacol. 118, 923 (1996). 